\title{
VASCULAR CHANGES AFTER INTERFERENCE WITH THE BLOOD FLOW OF THE FEMORAL HEAD OF THE RABBIT
}

\author{
André lemoine, Paris, France* \\ Formerly Girdlestone Scholar in Orthopaedic Surgery, Nuffield Orthopaedic Centre, Oxford
}

Despite repeated attempts to provide experimental data in support of the ischaemic theory of osteochondritis of the upper femoral epiphysis, decisive laboratory evidence on the etiology of the condition is still not available. It is possible that the difficulty in substantiating experimentally any of the numerous theories still in vogue is due to the rarity with which osteochondritis of the femoral head seems to occur in the animals used for experimental study, or in any other animal. It is not even clear whether the changes reported by Thomasen (1939) in the shoulder of the Danish pig are comparable with those affecting the upper femoral epiphysis of young children.

Many investigators have assumed that osteochondritis of the femoral head is caused by interference with the blood flow to the secondary nucleus of ossification, and have attempted to reproduce in animals, usually the rabbit or the dog, the changes that are seen in children. Nussbaum (1923) excised the whole of the synovial membrane of the hip and divided the ligamentum teres in dogs, and found changes which he thought were like those of osteochondritis of the hip. Bentzon (1927) injected alcohol near the epiphysial arteries of the hips of rabbits and obtained in one-sixth of them changes which he likened to those of juvenile osteochondritis. Introzzi (see Leriche 1934) also injected alcohol around the femoral neck of rabbits and concluded that he had produced "coxa plana" in a number of them, but Miltner and $\mathrm{Hu}$ (1933) repeated in rabbits and dogs Bentzon's and Introzzi's experiments and were unable to confirm them. On the other hand, Zemansky and Lippmann (1929) divided the ligamentum teres and part of the hip capsule of young rabbits and found that flattening of the femoral head occurred afterwards. Kistler $(1934,1935,1936)$ found that the femoral heads of rabbits could be altered in a way resembling human osteochondritis by several procedures: but Stewart (1933) divided the capsular attachment to the femoral neck in rabbits, Burrows (1941) and Lacroix (1942) divided the round ligament in rabbits, and Graham (1930) in goats. and all were able to produce changes similar to-but in their opinion not identical with-those of "coxa plana."

It appears from this short account of some of the experimental work on this subject that the interpretation of results varies more with the individual worker than with the methods used. It is noticeable that all these works, though describing the histological effects, are conspicuous for their uniform lack of information of the accompanying changes in vascular pattern. This consideration suggested that, with some of the more reliable methods of vascular investigation now available, it would be possible to study experimentally the changes in the vascular anatomy of the upper femoral epiphysis which follow interruption of the arterial blood flow.

\section{MATERIAL AND METHODS}

Sixty male and female rabbits of different ages, including a few adults, were used: but most were between the ages of two and seven weeks. Only the left hip was used in each animal, the right being left untouched as a control. In a few rabbits the distal femoral epiphysis had its blood flow interfered with as it had been noted that the main supply to this region came from a central posterior artery which could be easily divided.

* At present attached to the Department of Professor R. Merle d'Aubigné, Hôpital Cochin, Paris. 
All the animals were operated upon under general anaesthesia with nembutal, ether and trilene. Weekly radiographs of both femora were obtained from just before the time the experiment began until the animal was killed, which was after a period varying from four days to four months. Under nembutal all the animals had a cannula placed in the abdominal aorta and several substances of varying molecule or particle size were perfused through the cannula before they were killed. The most useful injection mass was a warmed mixture of one part of a 1 per cent solution of Berlin Blue with two parts of fine barium suspension (Micropaque). The amount of the injection was approximately in millilitres half the weight of

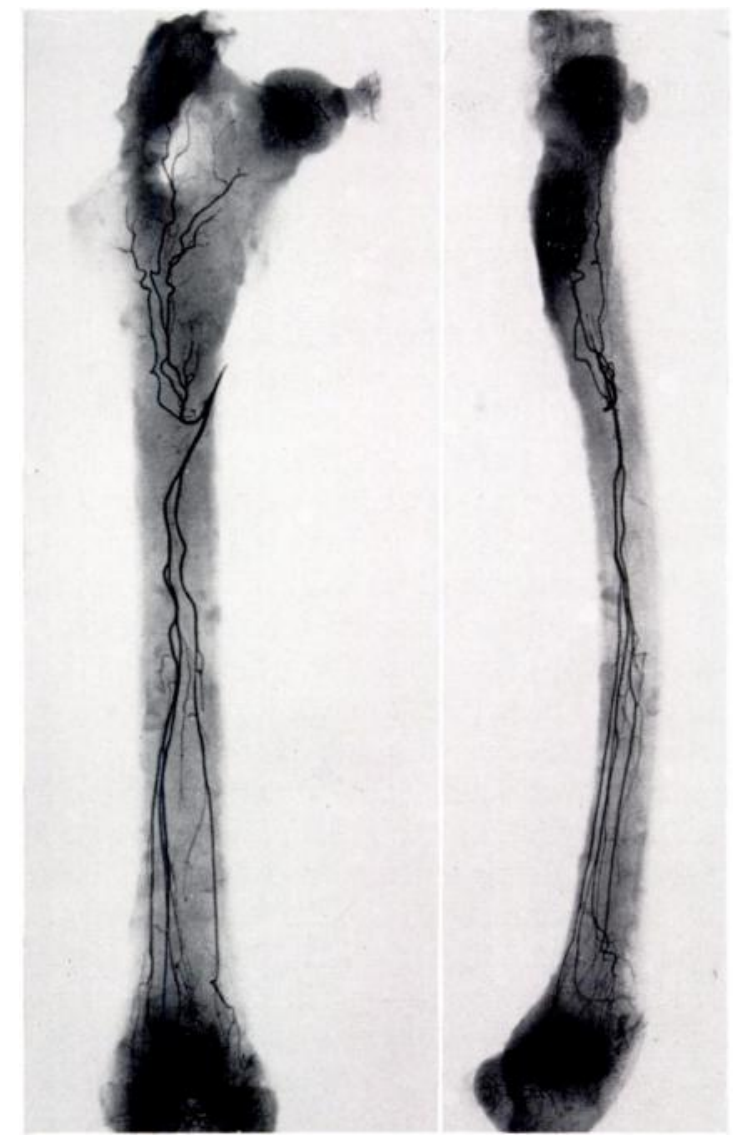

FIG. 1

Antero-posterior and lateral angiographs of the femur of a normal rabbit.

the animal in hectogrammes. The method of injection was that described by Trueta, Barclay Daniel, Franklin and Prichard (1947) adapted for the visualisation of the vessels within the bone tissue (Trueta and Harrison 1953). After injection the specimens were fixed in 10 per cent formalin. Microradiographs were obtained with the technique described by Barclay (195I). Photographs of the specimens prepared by the Spalteholz method were also obtained in a number of experiments.

The normal vascular anatomy of the upper femoral epiphysis of the rabbit-Before analysing the results of this investigation it seems convenient to describe the normal vascular anatomy of the proximal femoral epiphysis of the rabbit, because some features differ from those found in man (Trueta and Harrison 1953, Trueta 1957). 
As in man, the vascular pattern changes with growth and must always be studied in relation to age.

The main upper branches of the nutrient artery, both in the adult and young animal, vary in number from about three to six. The nutrient artery penetrates the femur where the epiphysis joins the shaft. On reaching the marrow cavity the nutrient artery divides into an ascending and a main descending branch (Fig. 1). The ascending branch gives origin to the large arterioles which distribute themselves throughout the whole of the upper metaphysis and anastomose with some of the vessels of the femoral head.

The terminal branches of the lateral circumflex artery-On reaching the narrow space between the lower border of the acetabulum and the upper aspect of the lesser trochanter-which is very large in the rabbit-this artery divides. The main terminal branch emerging from the anterior circumflex runs over the front of the femoral neck close to the capsular insertion and

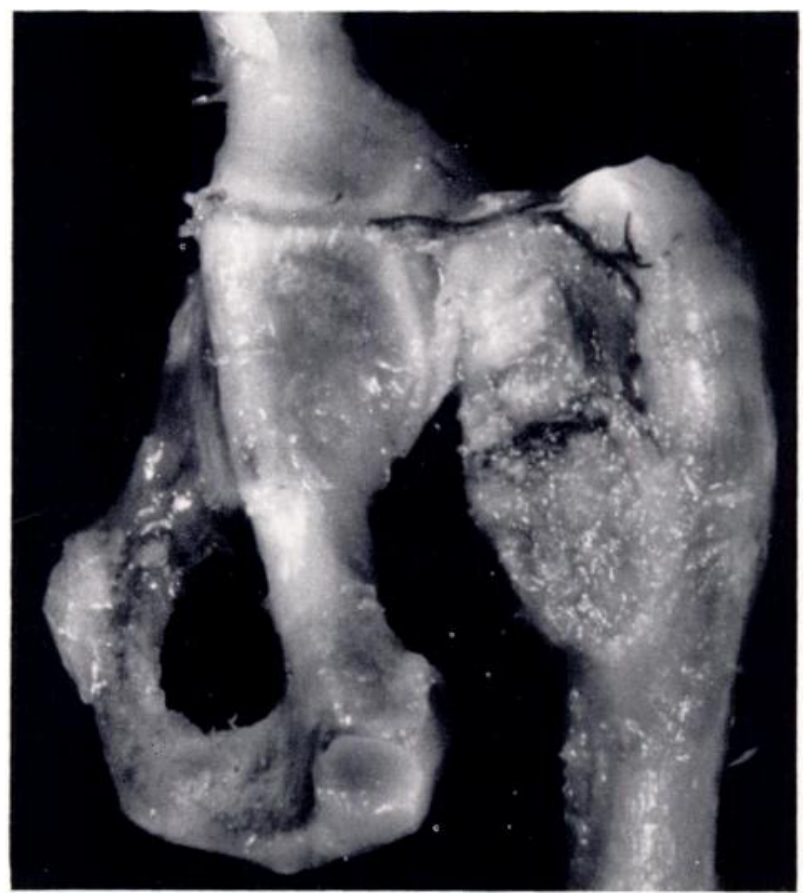

FIG. 2

Posterior view of the hip joint of the rabbit after injection into the abdominal aorta. Note the anastomosis between the sciatic artery and the main terminal branch of the anterior circumflex artery.

ends by anastomosing with a branch of the sciatic artery (Fig. 2). During its course this vessel gives origin to a number of capsular arterioles at the upper part of the joint (Fig. 3). This arrangement is present at all ages.

Another terminal branch of the anterior circumflex artery runs at all ages under the transverse acetabular ligament and anastomoses with branches of the obturator artery, contributing to the blood flow through the round ligament.

Finally, a third terminal branch of the anterior circumflex artery extends round the inferior aspect of the femoral neck. penetrating the postero-inferior insertion of the capsule and, after running under the synovial membrane for a few millimetres, enters deep into the postero-inferior part of the femoral head (Fig. 4). In young animals the point of entrance of this vessel is at the junction of the epiphysial cartilage with the neck. This vessel, a true 

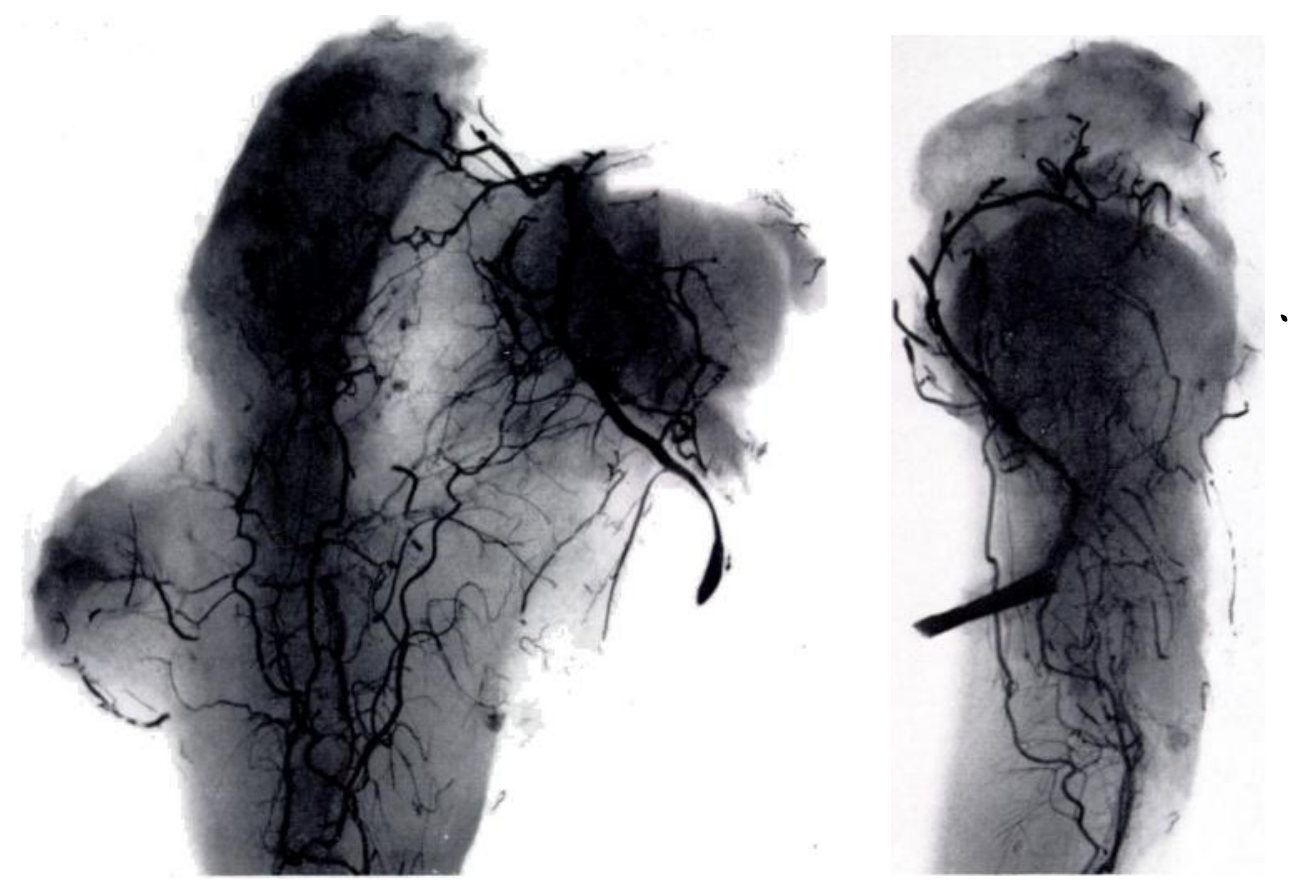

FIG. 3

Antero-posterior and lateral angiographs of the proximal end of an adult normal rabbit's femur. Note the capsular vessels and the large artery in front of the joint.
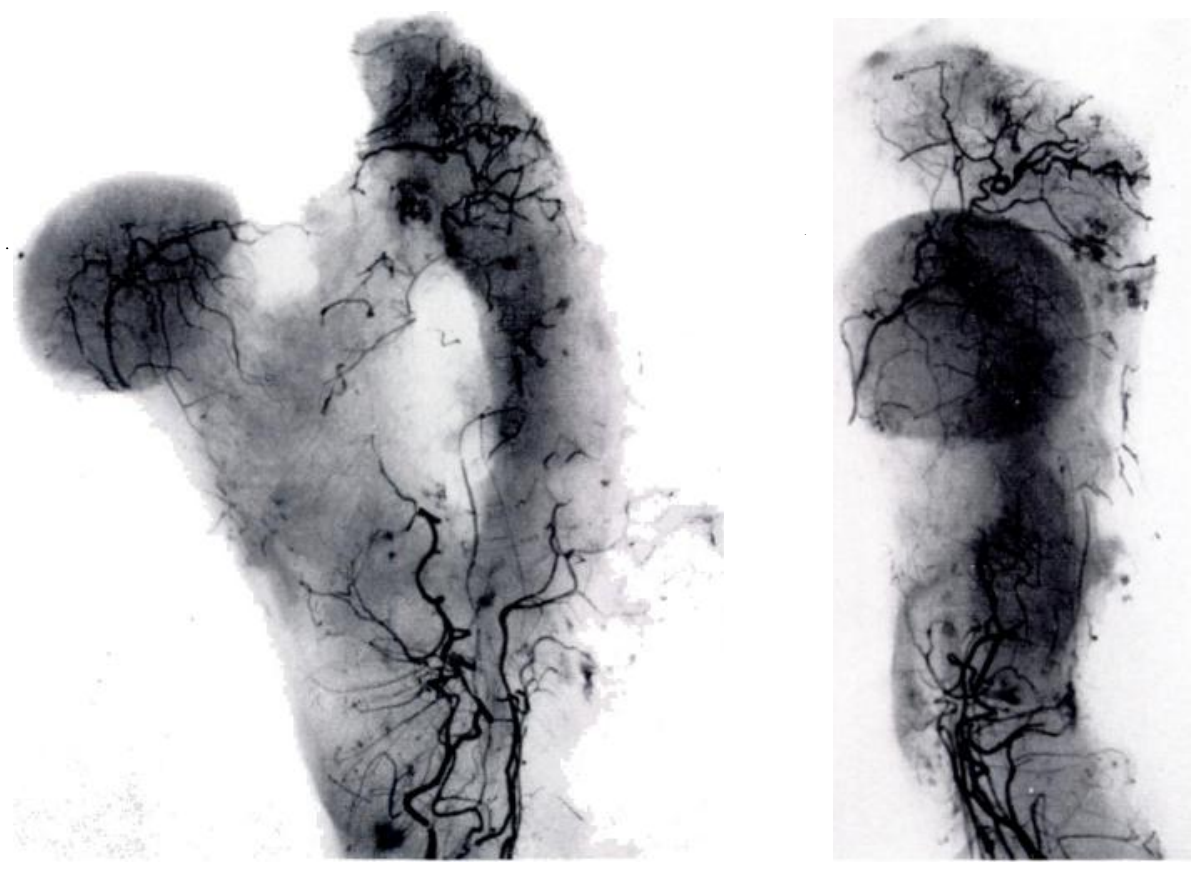

FIG. 4

Angiographs of the proximal end of a normal adult rabbit's femur showing the course of the "nutrient " artery to the femoral head. 
nutrient artery to the head, has no equivalent in man even if it resembles one of the metaphysial ascending arteries seen in early infancy (Trueta 1957). The variation that this artery undergoes during growth is caused by the fusion of the epiphysis: at about three months it anastomoses profusely with the vessels of the neck.

Perforating metaphysial arteries-These arteries vary in number and size but never reach the importance of those seen in children. They anastomose with the nutrient and the circumflex systems of vessels. Age does not seem to influence their development.

Vessels of the ligamentum teres-These vessels are greatly subjected to the changes of age. like the corresponding arteries in man.

In the three-day-old rabbit a sizeable vessel enters the head from the round ligament and anastomoses with the ascending branch of the circumflex artery which I have named the nutrient artery of the head.

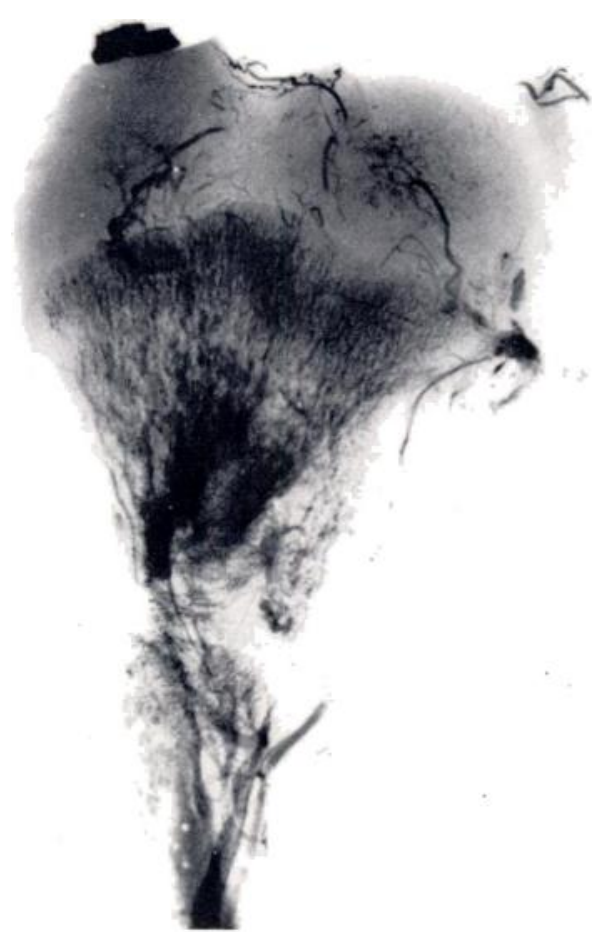

Fici. 5

Antero-posterior angiograph of the proximal end of the normal femur of a seventeen-day-old rabbit. The " nutrient " artery to the head is seen: the arterioles of the ligamentum teres do not penetrate any longer towards the epiphysial ossicle.

At nine days the arteriole of the round ligament is still present but it is already decreasing in importance.

At seventeen days it has ceased to contribute to the nourishment of the secondary nucleus of ossification and when present does not penetrate the head (Fig. 5).

These observations are at variance with those of Zemansky and Lippmann (1929), who demonstrated by arterial injection that the vessels of the ligamentum teres supply a considerable part of the epiphysial nucleus of the rabbit at two weeks, a much smaller part at five weeks and fail to reach the nucleus at seven weeks. Burrows (personal communication) has observed vessels of the ligamentum teres penetrating into the nucleus in rabbits aged twenty-two to twenty-nine days.

VOL. 39 B. NO. 4, NOVEMBER 1957 


\section{RESULTS}

The results obtained in the present investigation have been grouped according to the type of vascular interference which had been caused to the upper femoral epiphysis. In a separate short section the results obtained by causing vascular damage to the distal femoral epiphysis will be reported with the aim of comparing them with those found in the proximal epiphysis.

\section{EXPERIMENT 1. LIGATURE OF THE LIGAMENTUM TERES (SEVEN RABBITS)}

Through a postero-lateral incision the fibres of gluteus maximus were split and the tendons of the medius and minimus were divided near their insertion to the greater trochanter. The upper part of the capsule was then incised and a fine silk ligature was placed round the ligamentum teres by dislocating the hip.

Results-Five rabbits from two to three weeks old were used. One rabbit was killed on each of the tenth, twenty-first, twenty-eighth, thirty-fifth and sixty-fifth days. All of the first four

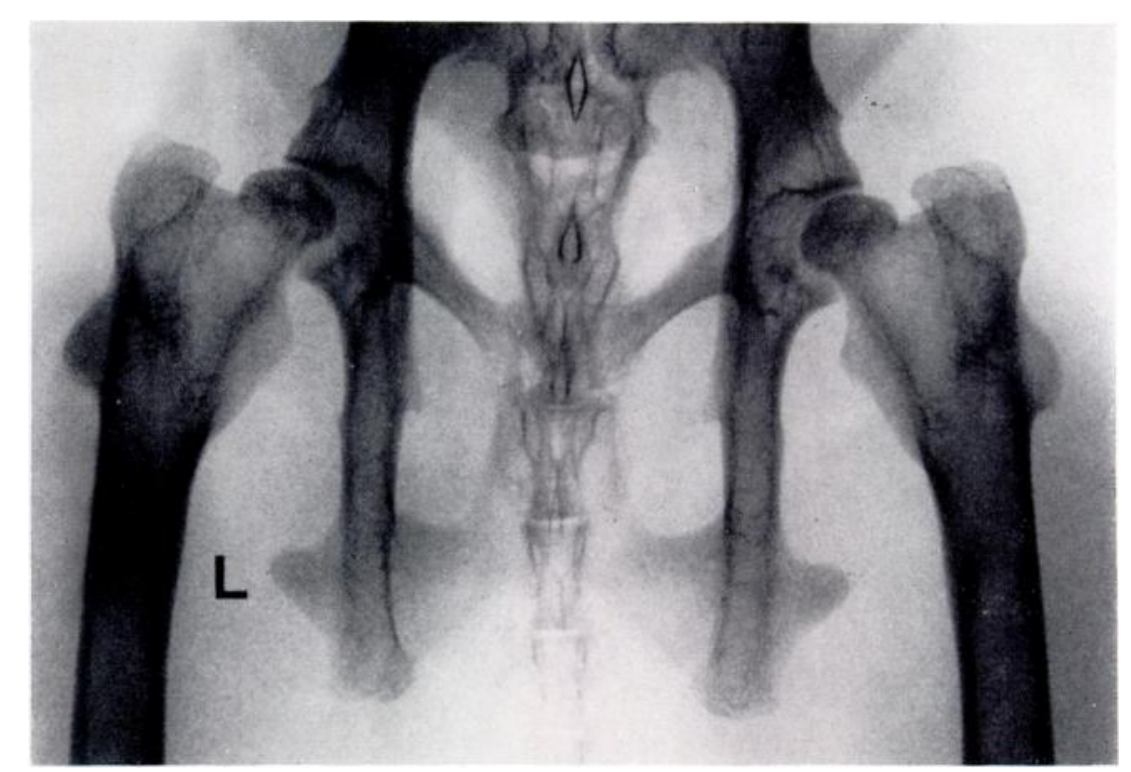

FIG. 6

Fragmentation of the left femoral head twenty-one days after the operation described under Experiment 1.

rabbits showed fragmentation of the epiphysis and flattening of the head (Fig. 6). The femoral neck appeared short and thickened (Figs. 7 and 8) and micro-angiographs showed great increase in vascularity (Fig. 9) in all but the first. In the fifth rabbit no change was seen in head or neck. (The " nutrient" vessel to the head was seen to be intact.) Two adult rabbits were killed at the fiftieth day and no change was found in the femoral head.

Comment-These results must be viewed with some caution. To ligate the round ligament a relatively large capsulotomy is necessary and a variable amount of damage to the capsular vessels is unavoidable. Moreover, to pass the needle round the ligamentum teres the femoral head must be pulled out of the acetabulum and even then the needle may cause some damage to the inner side of the capsule or to the joint cartilage. Finally, the silk is a foreign body which may cause some reaction. Further rabbits were operated on in an attempt to eliminate some of the misleading factors. 


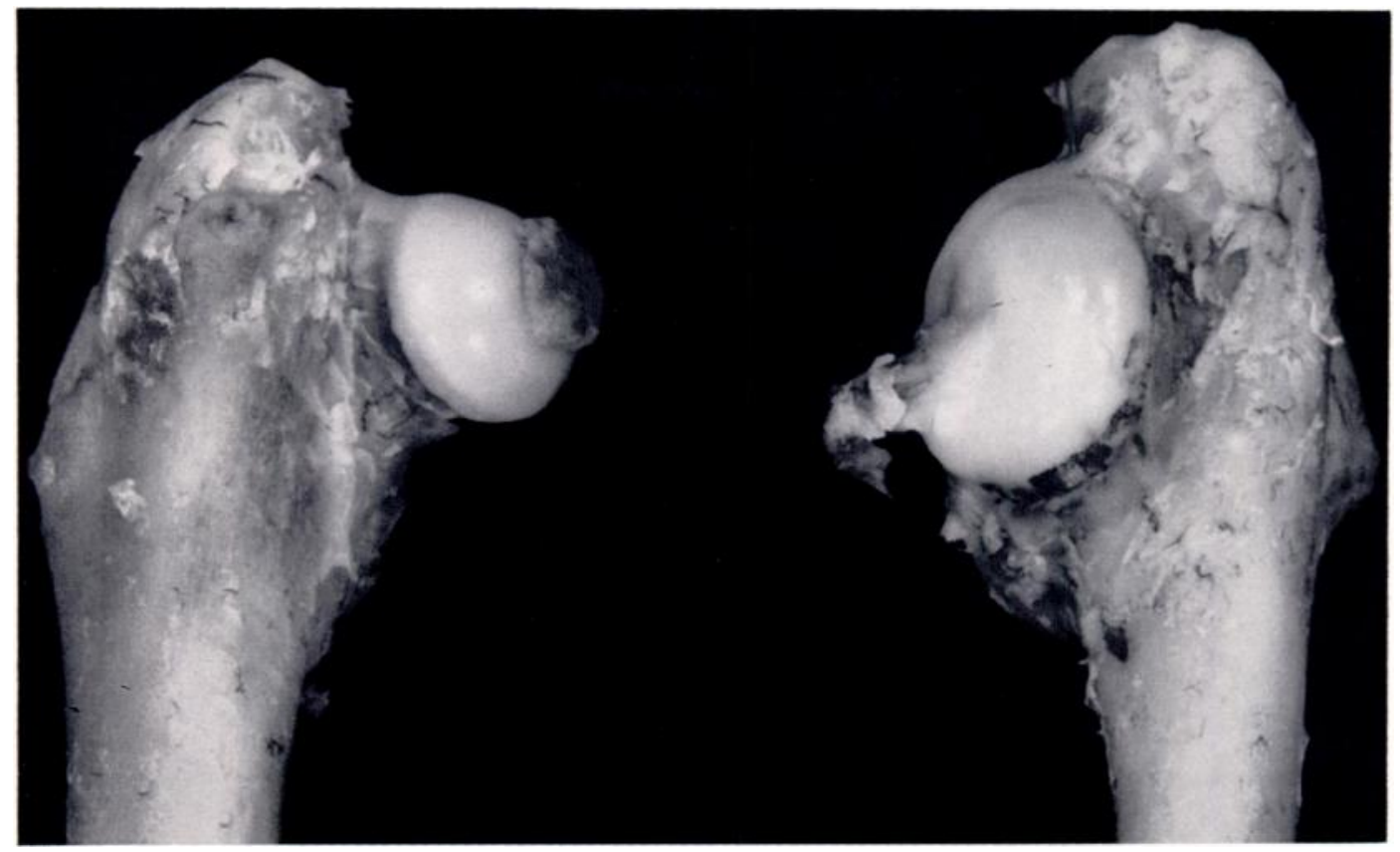

FIG. 7

Photograph of the anterior aspect of the proximal femoral end of a nineteen-day-old rabbit after the operation described under Experiment 1. The control side is shown on the left and the side operated upon on the right. Note the marked flattening of the head.

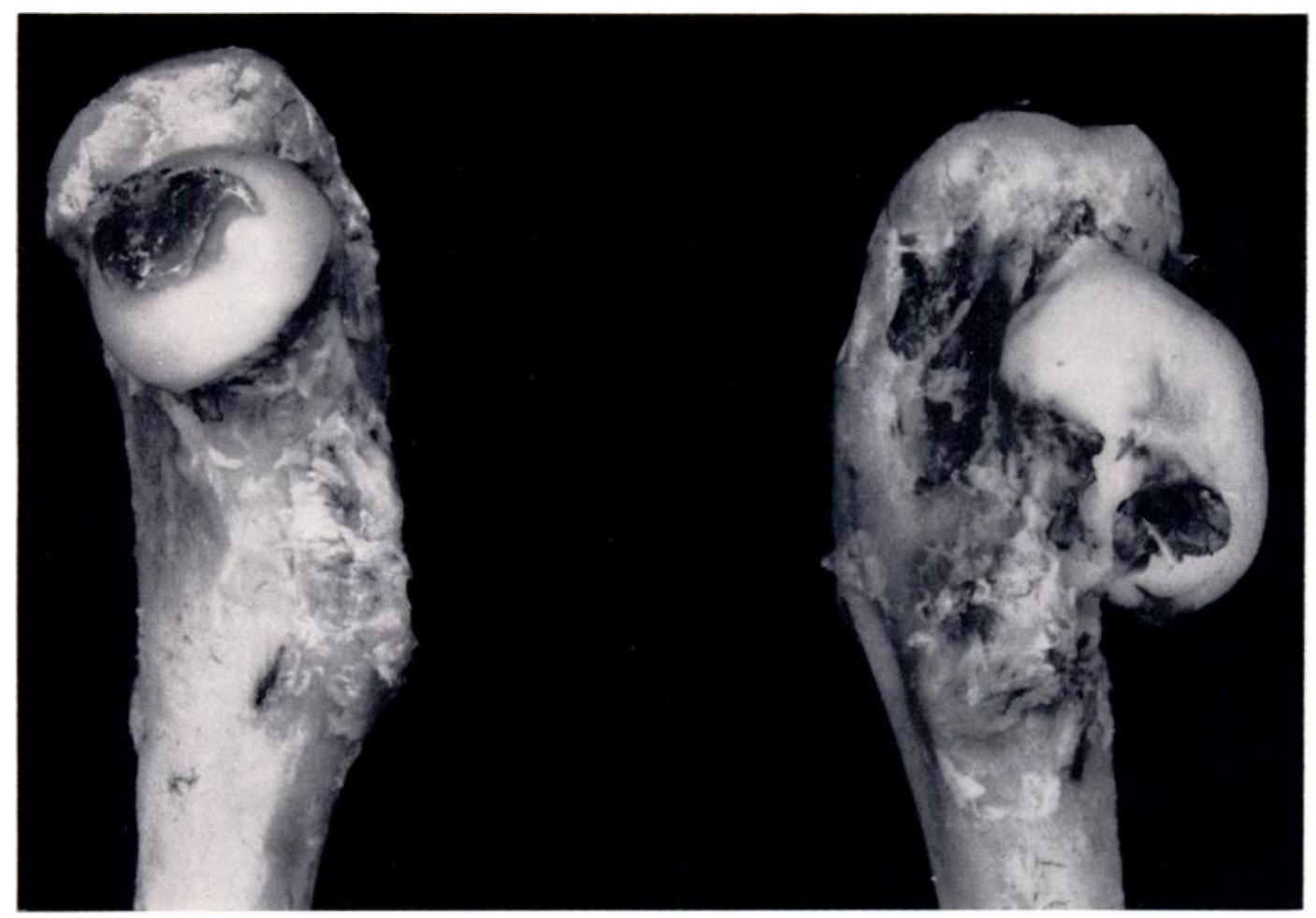

FIG. 8

Photograph of the medial aspect of the same specimen as shown in Figure 7. Note the extreme anteversion of the femoral neck on the side operated upon (right).

VOL. 39 B, NO. 4, NOVEMBER 1957

L 


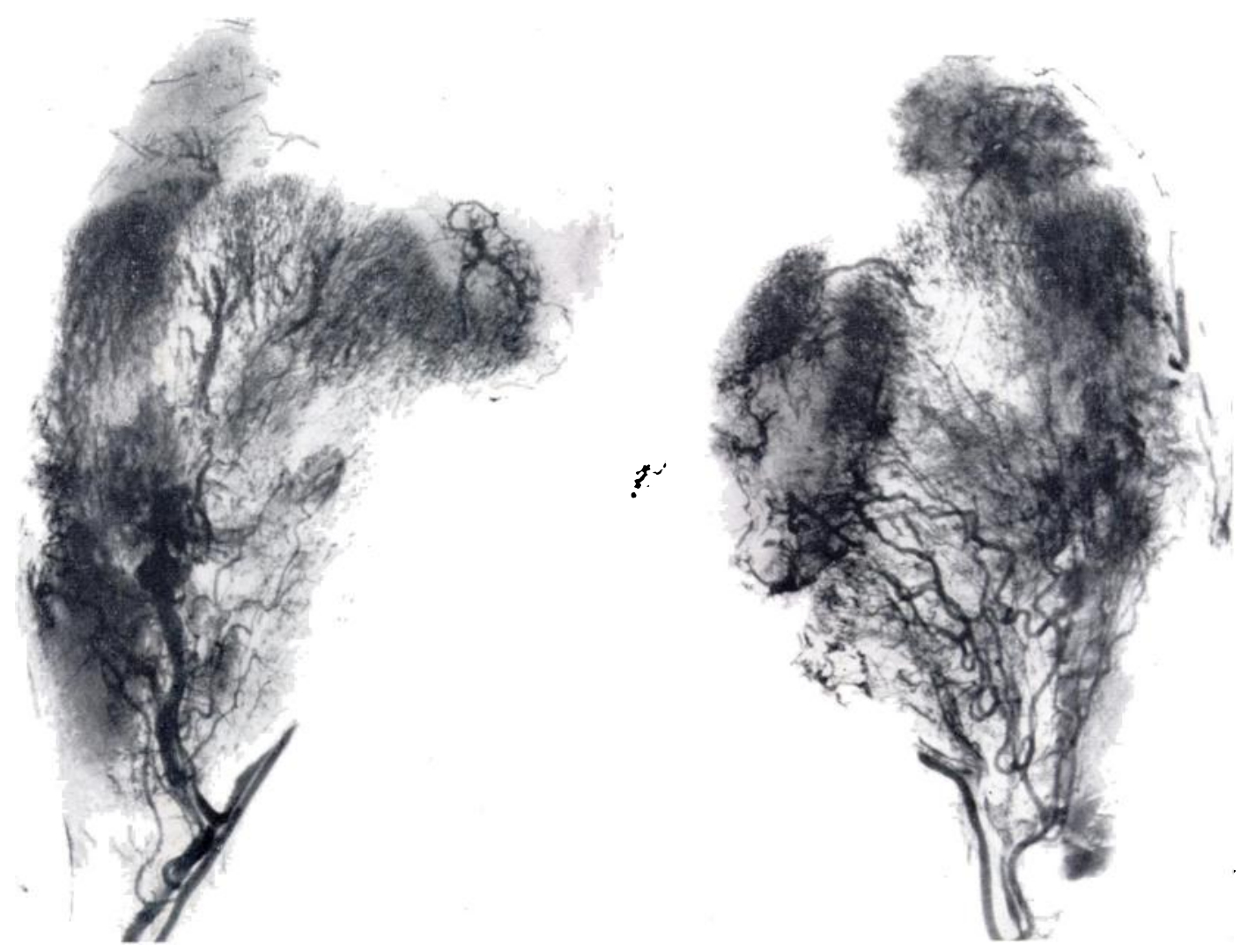

FIG. 9

Antero-posterior angiograph corresponding to the preceding specimen. Note the considerable increase in the size and number of vessels in the experimental side (right).

EXPERIMENT 2. DIVISION OF “ NUTRIENT" VESSEL TO THE HEAD

By a postero-lateral approach to the hip, splitting the gluteus maximus and dividing the tendons of the medius and minimus and of the pyramidalis near the trochanter, the capsule was divided in its lower part and the artery divided at its entrance into the head.

Results-The sixteen young rabbits were killed between the sixth and the eighty-fifth day after operation. Every animal showed some changes detectable by radiography, histology or macroscopically, such as flattening of the femoral head and shortening of the neck, as detailed below.

Rabbit 55-Operated upon when sixteen days old. Two days later the radiograph showed some blurring of the upper femoral extremity. The animal was killed on the sixth day. The epiphysial nucleus was smaller than that of the opposite (control) side; the femoral neck was shorter than normal and in varus, and the head was flattened. Angiography showed a marked increase in vascularity of the neck and greater trochanter. There was absence of injection mass in the area of distribution of the " nutrient" artery of the head. The secondary nucleus of ossification appeared denser in the radiographs and less well vascularised than the normal, and it was injected only through a small vessel from the round ligament (Fig. 10).

Rabbit 49-Operated on when eighteen days old. The radiographs taken fifteen days later showed a slight flattening of the femoral head and osteoporosis of the upper part of the femur. Micro-angiography showed a marked hypervascularisation of the head sprouting from the vessels of the ligamentum teres and the ascending (" nutrient") vessel (Fig. 11). 
Rabbit 44-Operated on when twenty days old. Twenty-three days later the radiographs showed a flattened head, smaller than on the control side. The neck was varus. Microradiographs obtained on the same day gave clear evidence of the great hypervascularisation of the femoral head, the majority of new vessels emerging from the ascending metaphysial artery (Fig. 12). Rabbit 53-Operated on when nineteen days old. Thirty-three days later the radiographs showed marked osteoporosis and flattening of the femoral head with enlargement of the superior aspect. The photographs gave clear evidence of these findings (as in Figs. 6 and 7). The microradiograph showed a better arterial injection of the side operated upon, but the increase in vascularity was less marked than in the previous rabbit injected two weeks sooner after operation (Fig. 13).

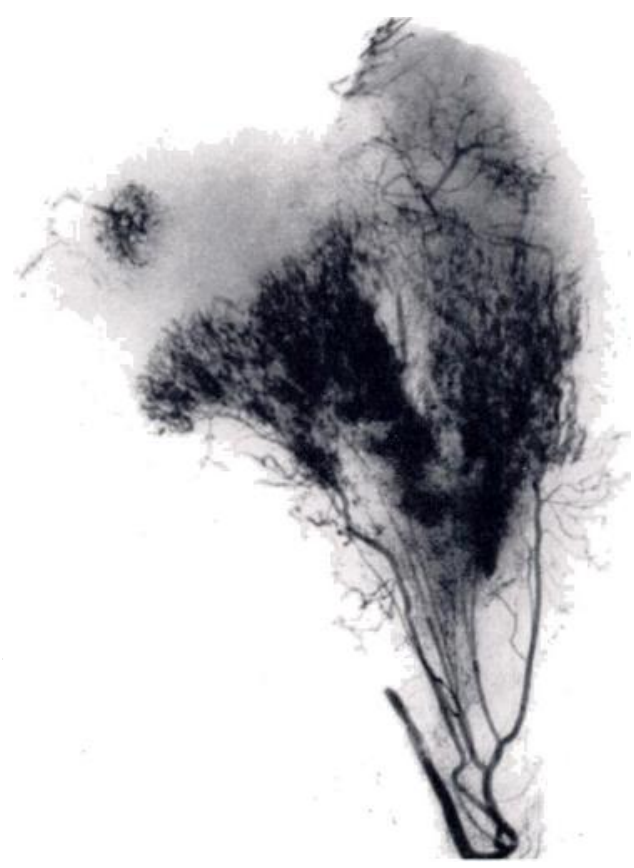

Fig. 10

Angiograph taken six days after the operation described under Experiment 2 , in a rabbit sixteen days old. It shows the decrease in vascularity of the secondary nucleus of ossification of the femoral head, the injection mass having reached the ossicle through the artery of the ligamentum teres, which on the control side was already undetectable.

Rabbit 59-Operated on when twenty-eight days old. Ten days later the radiograph showed porosis of the upper end of the femur, and the epiphysial ossicle was smaller than on the control side. On the seventeenth day the head appeared flattened and small in the radiograph. On the twenty-fourth day the secondary nucleus of ossification appeared fragmented and flattened and the neck was in marked varus. On the thirtieth day the head was still flattened but the structure of the epiphysial ossicle was becoming normal although the neck was still in varus. On the thirty-seventh day the head was flattened and of irregular density. The growth of the greater trochanter had been accelerated and it appeared at a higher level than that of the control side. The neck was shorter and varus (Fig. 14). On the fifty-fourth day the flattened head was tending to subluxate. On the eighty-fifth day the head remained denser than the control and subluxated. Fusion of the epiphysial cartilage, which was well advanced on the control side, was still in the early stages on the side of operation.

VOL. 39 B, NO. 4, NOVEMBER 1957 


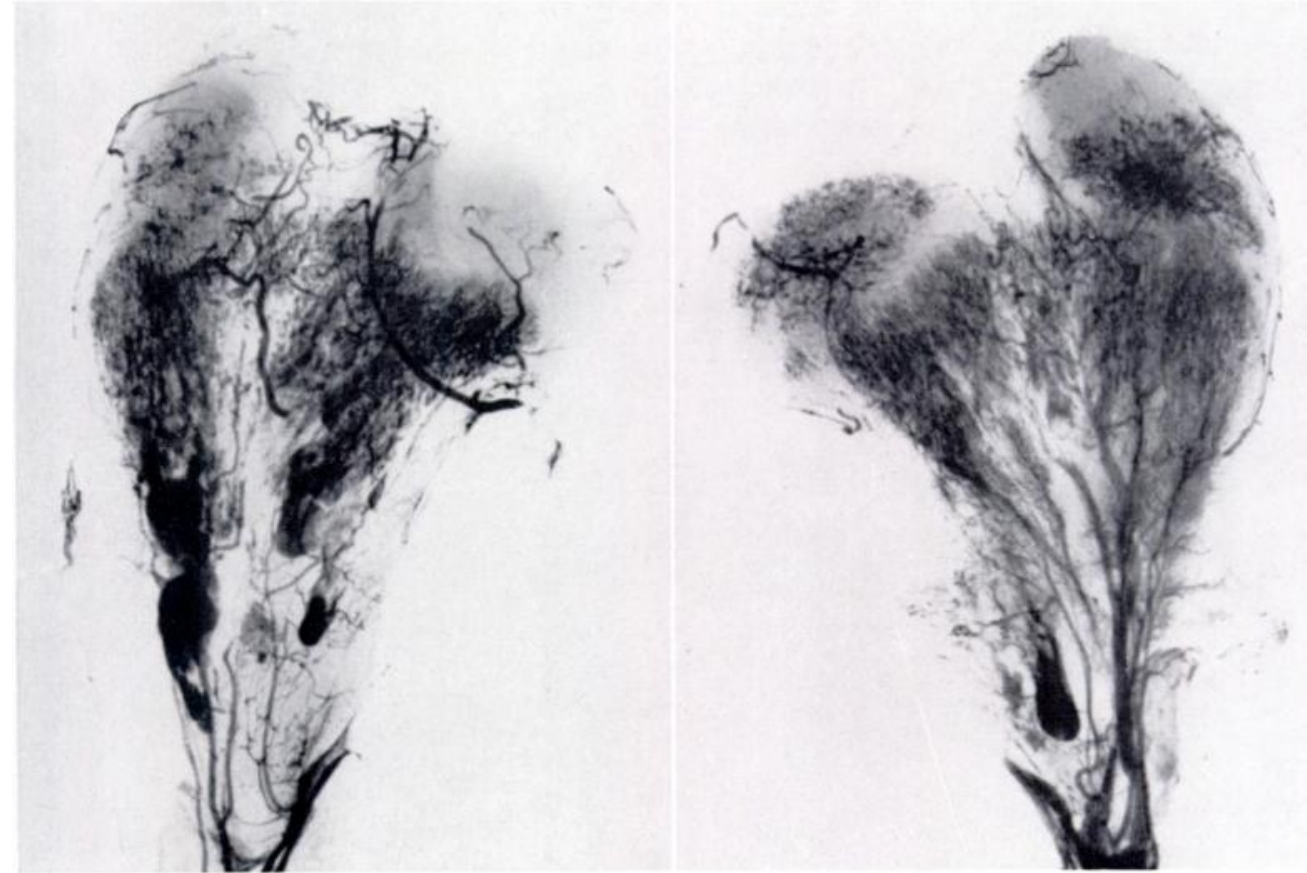

FIG. 11

Angiographs of a thirty-three-day-old rabbit which had the " nutrient " artery of the femoral head divided fifteen days before. The experimental side (at right) shows the flattening of the head and its profuse vascularity. The main source of blood is the ligamentum teres, apart from some contribution from the metaphysial vessels which cross superficially to the epiphysial cartilage.

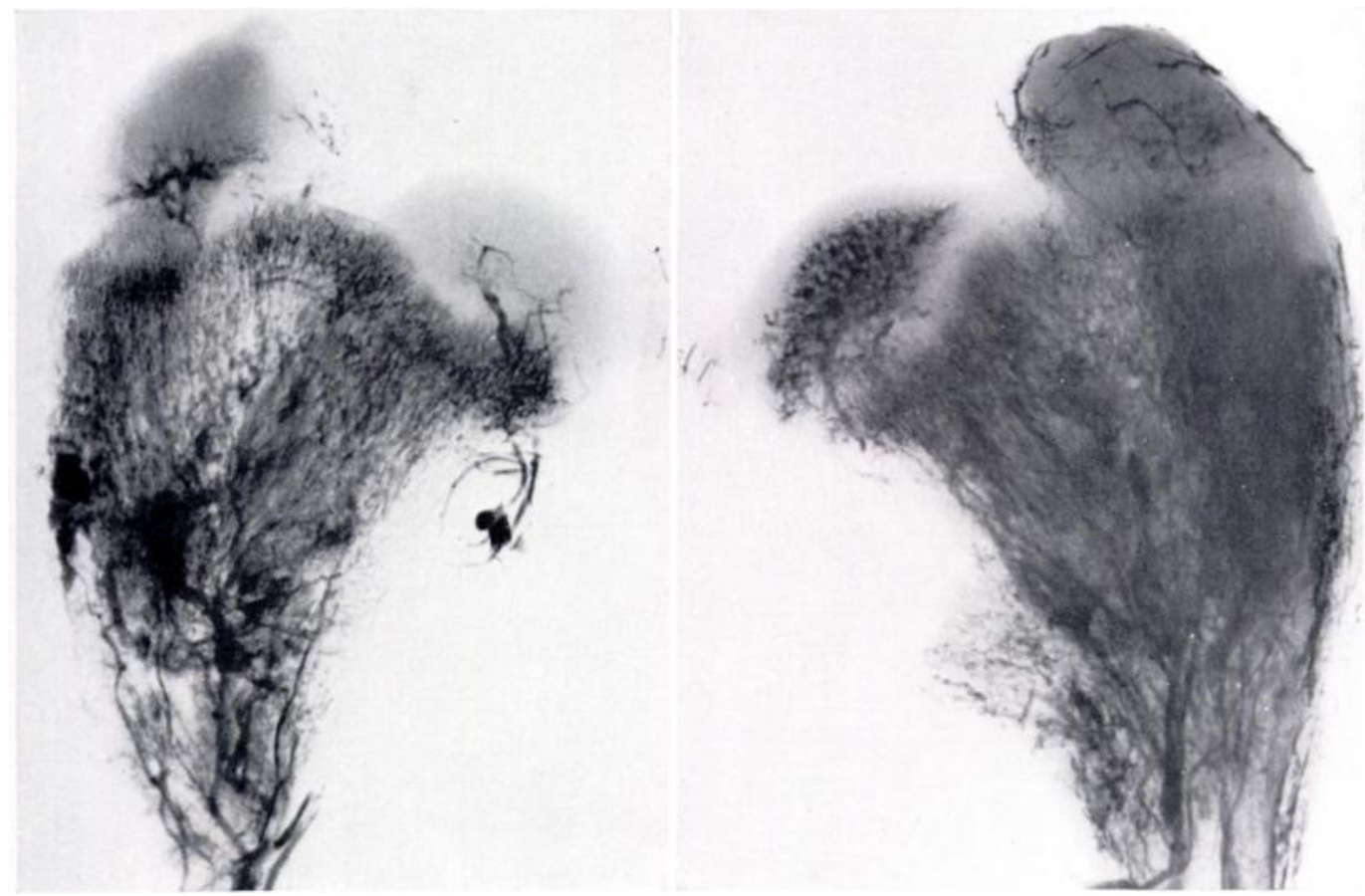

Fig. 12

Angiographs of a forty-three-day-old rabbit, twenty-three days after the operation described in Experiment 2. The femoral head shows very considerable increase in vascularity. The control side is shown at left. 


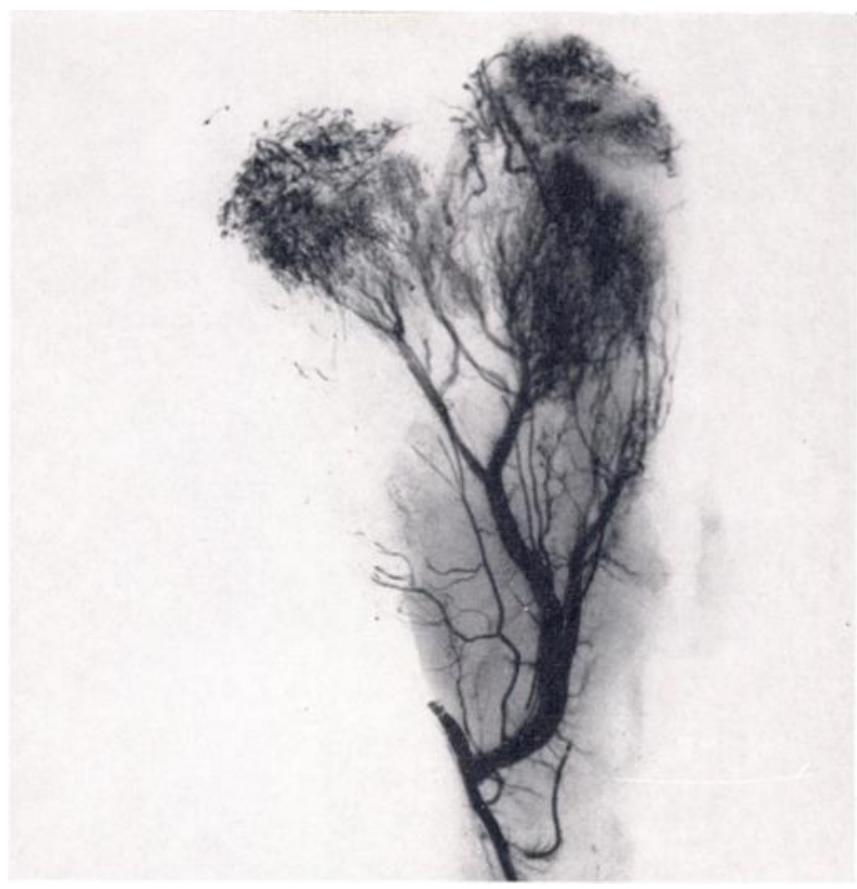

FICi. 13

Angiograph of a fifty-four-day-old rabbit operated upon when fifteen days old. The vascularity of the femoral head is less profuse than in the preceding rabbits injected and killed after shorter periods.

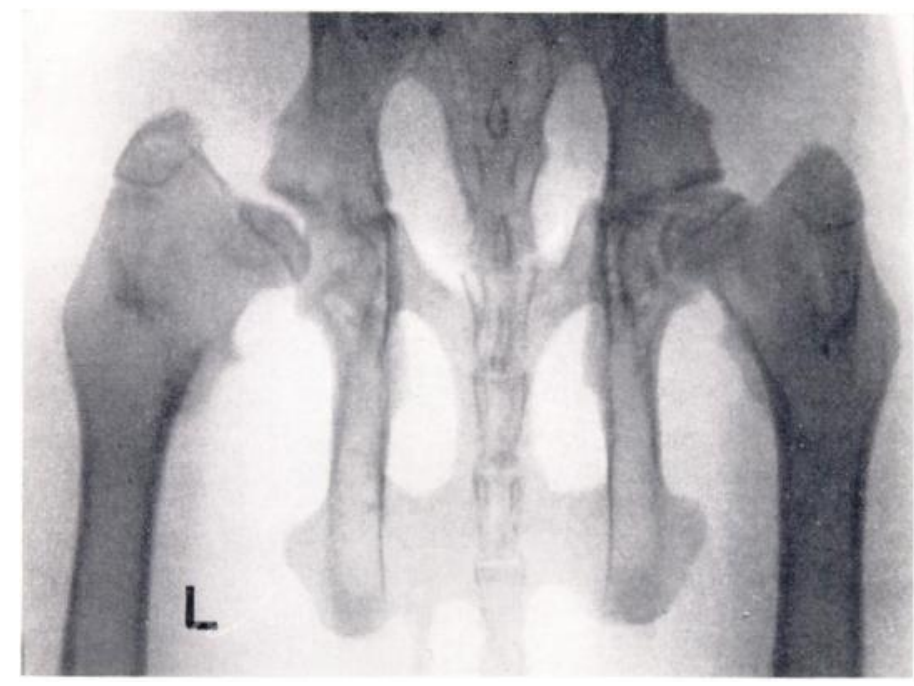

Fici. 14

Radiograph obtained thirty-seven days after division of the " nutrient " artery of the femoral head $(/ e f t)$ in a rabbit twenty-eight days old. Note the flattening of the femoral head and the shortening and broadening of the neck. 
The microradiographs at that time show that the vascularisation of the femoral head was like that of the control side but depended more on the vessels of the ligamentum teres for its blood flow than on the normal side (Fig. 15).

\section{EXPERIMENT 3. DIVISION OF THE ANTERIOR CIRCUMFLEX ARTERY}

This vessel was divided on the front of the femoral neck after the fibres of the gluteus maximus had been split and the tendon of the medius and minimus detached. This series of rabbits was grouped by age.

Results-Eight rabbits eighteen days old were killed from the thirty-fifth day to the hundredth day. Only a very slight tendency to flattening of the head was noted. No changes were seen in the angiographs.

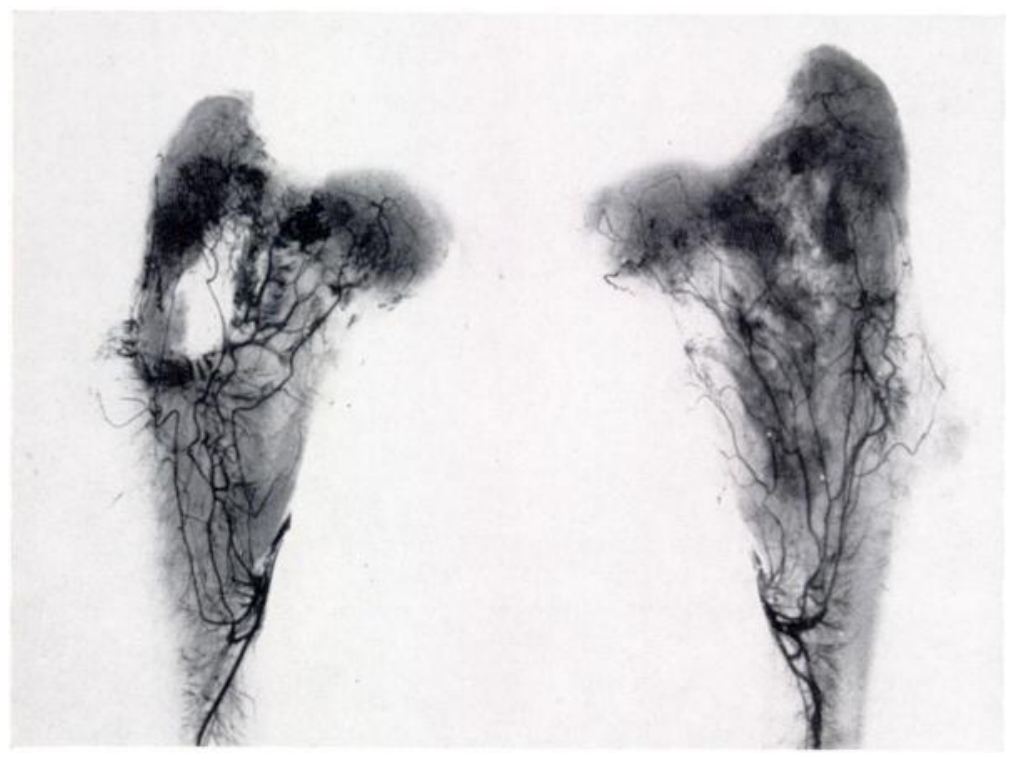

FIG. 15

Angiographs of the rabbit in the preceding figure, injected eighty-five days after the operation. The vascularity of both femoral heads is very similar, but on the side of the operation (left) the blood supply is derived from the vessels of the round ligament and those of the metaphysis.

Six rabbits thirty-five days old were killed from the fourteenth to the ninetieth day. The femoral head appeared very slightly flattened. No change was noted in the angiographs.

Three adult rabbits were killed at the thirty-fifth, forty-second and ninetieth days. No lesion of any kind was seen.

It was thus demonstrated that the division of the circumflex artery, whatever the age of the animal, had hardly any effect upon the femoral head.

\section{DISCUSSION}

The radiographic changes appearing in the capital femoral ossicle after division of the large metaphysial branch from the anterior circumflex, which I refer to as the "nutrient" artery of the femoral head, resemble those of human juvenile osteochondritis both in their characteristics and in their evolution towards spontaneous healing, with a variable amount of permanent deformity of the head and neck of the femur.

The process by which the changes of the femoral head became established in the experimental animals was as follows. Immediately after division of the "nutrient " capital artery there was ischaemia of the ossicle, but by the eighth day revascularisation began 
through the round ligament and was followed by revascularisation in the area supplied by the inferior metaphysial arteries. About the twelfth day a generalised decrease in vascularity was still detectable. Radiographically, this first anaemic stage corresponded to an increase of bone density together with a decrease in size of the secondary nucleus of ossification. After these early changes the vascularity began to increase about the fifteenth day, with the development of vessels which had already entered the head through the above mentioned routes. This vascular profusion was accompanied by radiographic signs of bone reabsorption. At this stage the experimental animals showed great restriction of muscle and joint activity from pain and muscle spasm. The bone and articular deformity, consisting of flattening of

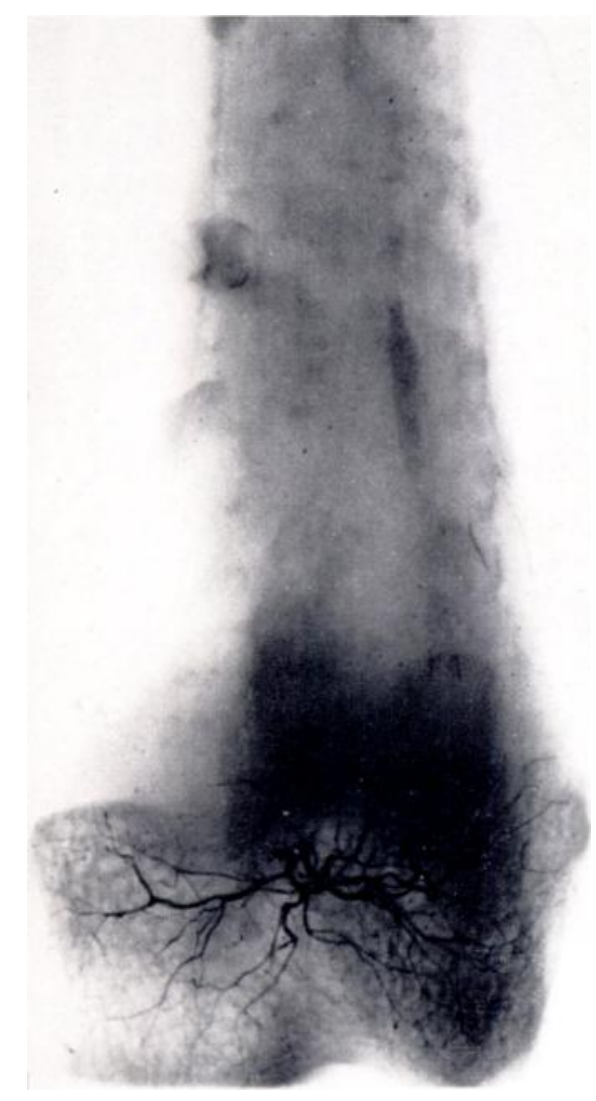

FIG. 16

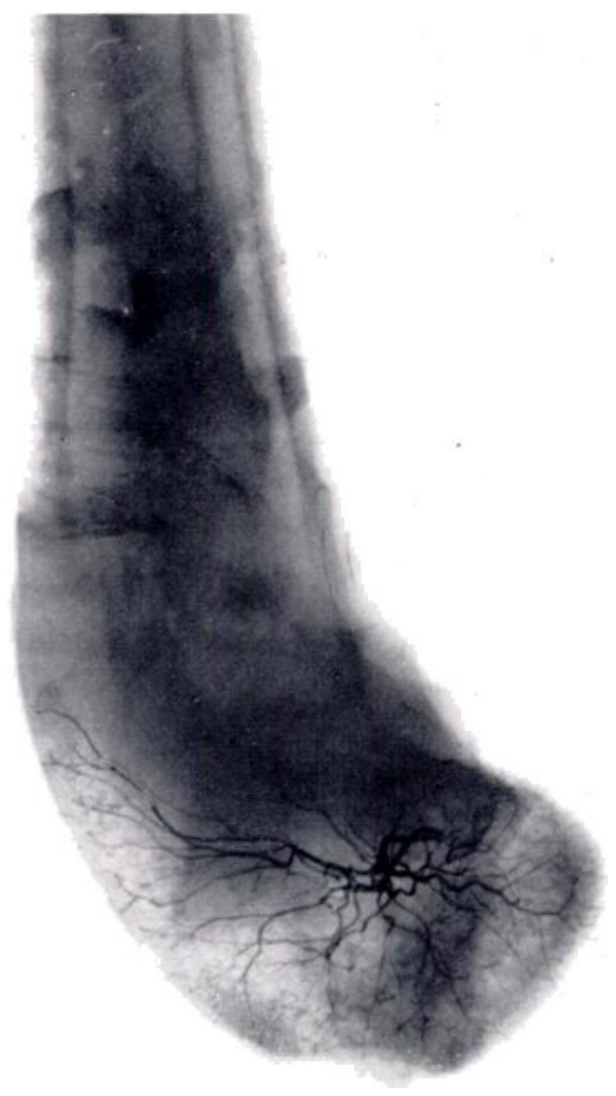

Antero-posterior and lateral arteriographs of the distal femoral epiphysis of an adult rabbit showing the large and single epiphysial artery entering the bone from the back.

the femoral head and inclination of the neck in varus and anteversion, appeared during this phase of the post-ischaemic process.

About the ninetieth day after operation the hyperaemia ceased and the experimental disease may be considered to have ended but it left a permanently deformed hip joint and a prematurely fused epiphysial cartilage.

\section{EXPERIMENTS UPON THE DISTAL FEMORAL EPIPHYSIS}

In the course of studies in this laboratory on the vascular patterns of bones it has been observed that the distal femoral epiphysis of the rabbit is mainly supplied by a large branch from the popliteal artery which is situated near the centre and back of the intercondylar notch. 
After passing underneath the capsule it penetrates the bone at the proximal aspect of the epiphysis, and from there is expanded in all directions without crossing the barrier of the epiphysial plate up to three months of age. It anastomoses in young rabbits with periosteal and perforating vessels which penetrate the bone at different points of the capsular insertion (Fig. 16). In adult rabbits this central epiphysial vessel anastomoses freely with the terminal branches of the nutrient artery.

Since this artery supplies the main blood volume to the distal femoral epiphysis, it was decided to investigate whether changes could be produced in the epiphysis by its division. In seven rabbits the vessel was exposed by a medial longitudinal incision and divided.

Four adult rabbits were killed at fifteen, forty-five, sixty-five and ninety days afterwards. No change in the epiphysis was noted, and the microradiographs were normal in all cases.

Three rabbits aged fourteen, twenty-one and thirty-five days were operated upon. The first and last animals showed a very slight shortening of the femur, together with an extremely discrete hypervascularisation. The rabbit operated on at twenty-one days was killed fourteen days later and showed a posterior slipping of the distal femoral epiphysis together with some hypervascularisation.

Comment-It is of interest that, in an epiphysis like the distal femoral epiphysis where the vessel supplying it anastomoses with a number of periosteal and perforating vessels during growth, no changes of ischaemic-hyperaemic nature were produced by the interruption of this main artery. This further supports the belief that the "osteochondritic " changes occurring in the upper femoral epiphysis after division of the main artery are dependent on the peculiar local anatomical arrangement which places the responsibility for the blood supply of the growing epiphysis on one large vessel or group of vessels.

\section{CONCLUSIONS}

1. By the surgical division of the main capsular artery supplying the upper femoral epiphysis of the rabbit it is possible to cause changes which resemble those occurring in human osteochondritis.

2. The phase of anaemia (ischaemia and hypovascularisation) lasts in the rabbit less than fifteen days. The whole process lasts approximately ninety days, and only for one-sixth of this period does the femoral head suffer from a reduction in its blood supply.

3. After the fifteenth day until the end of the process the condition changes to one of hypervascularisation, which lasts six times longer than that of ischaemia or relative anaemia. 4. By the ninetieth day the whole process has lost its activity and only some permanent deformities remain. The vascular pattern is from then on the normal in the rabbit.

5. The "osteochondritic" changes cannot be elicited in the distal femoral epiphysis. The apparent reason is the presence of anastomoses between the main artery and other epiphysial vessels.

6. There seems to be reasonable experimental evidence, by implication, in favour of the vascular theory of osteochondritis of the upper femoral epiphysis in children.

I must express my gratitude to Professor J. Trueta for his constant help and encouragement throughout this work. I would like to express my thanks to Professor R. Merle d'Aubigné for his advice. I am also grateful to Mr D. W. Charles and Mr A. H. Mann for their technical contribution.

\section{REFERENCES}

Barclay, A. E. (1951): Micro-Arteriography. Oxford: Blackwell Scientific Publications.

Bentzon, P. G. K. (1927): Roentgenological Investigations Concerning the Arterial Supply of the Epiphyses. Acta Radiologica, 8, 618.

Burrows, H. J. (1941): Coxa Plana, with Special Reference to its Pathology and Kinship. British Journal of Surgery, 29, 23.

Graham, R. V. (1930): Experimental Considerations in Perthes's Disease. Medical Journal of Australia, i, 207. 
KistLer, G. H. (1934): Sequences of Experimental Infarction of the Femur in Rabbits. Archives of Surgery, 29, 589.

KistLeR, G. H. (1935): Sequences of Experimental Bacterial Infarction of the Femur in Rabbits. Surgery, Gynecology and Obstetrics, 60, 913.

Kistler, G. H. (1936): Effects of Circulatory Disturbances on the Structure and Healing of Bone. Injuries of the Head of the Femur in Young Rabbits. Archives of Surgery, 33, 225.

LACRoIx, P. (1942): Les vaisseaux du ligament rond dans la pathogénie de la coxa plana. Revue d’Orthopédie, 28, 30.

LERICHE, R. (1934): Recherches expérimentales sur le mécanisme de formation de l'ostéochondrite de la hanche. Lyon Chirurgical, 31, 610.

Miltner, L. J., and Hu, C. H. (1933): Osteochondritis of the Head of the Femur. An Experimental Study. Archives of Surgery, 27, 645.

Nussbaum (1923): Demonstration über die Erzeugung von Osteochondritis juvenilis auf experimentellem Wege. Zentralblatt für Chirurgie, 50, 937.

Stewart, W. J. (1933): Aseptic Necrosis of the Head of the Femur Following Traumatic Dislocation of the Hip Joint. Journal of Bone and Joint Surgery, 15, 413.

Thomasen, E. (1939): Calvé-Perthes-like Changes in the Head of the Humerus in Swine. Acta Orthopaedica Scandinavica, 10, 331.

TruetA, J. (1957): The Normal Vascular Anatomy of the Human Femoral Head During Growth. Journal of Bone and Joint Surgery, 39-B, 358.

Trueta, J., Barclay, A. E., Daniel, P. M., Franklin, K. J., and Prichard, M. M. L. (1947): Studies of the Renal Circulation. Oxford: Blackwell Scientific Publications Ltd.

Trueta, J., and Harrison, M. H. M. (1953): The Normal Vascular Anatomy of the Femoral Head in Adult Man. Journal of Bone and Joint Surgery, 35-B, 442.

Zemansky, A. P., Jun., and LippmanN, R. K. (1929): The Importance of the Vessels in the Round Ligament to the Head of the Femur During the Period of Growth, and their Possible Relationship to Perthes' Disease. Surgery, Gynecology and Obstetrics, 48, 461. 